\title{
X-ray Microscopy and Spectroscopy Combine to Probe Selenium Biology.
}

\author{
Hugh H. Harris ${ }^{1 *}$, Melanie J. Ceko ${ }^{1}$, Claire M. Weekley ${ }^{1}$, Paul K. Witting ${ }^{2}$ and Raymond J Rodgers ${ }^{3}$ \\ 1. Department of Chemistry, The University of Adelaide, Adelaide, Australia. \\ 2. Discipline of Pathology, The University of Sydney, Sydney, Australia. \\ 3. Obstetrics and Gynaecology, The University of Adelaide, Adelaide, Australia. \\ * Corresponding author: hugh.harris@adelaide.edu.au
}

Selenium is essential for life; twenty-five proteins contain selenocysteine (SeCys) at their active site.[1] These selenoproteins are mostly enzymes involved in cellular redox reactions (reduction-oxidation reactions that result in electron transfer, which underpins fundamental enzyme function and cellular signalling), but also include Se storage and transport proteins.[2, 3] The full complement of selenoproteins (the selenoproteome) is critical to normal physiology, and the appropriate dietary Se intake required to establish a fully functional selenoproteome is very low. Although most modern communities are not Se-deficient, appreciable geographical variation exists,[4] usually based on local soil Se content.

A low Se status has been found to coincide with a higher incidence of various diseases ${ }^{[4]}$, likely due to dysfunction of core selenoproteins, [5] leading to a high incidence of dietary supplementation with Se. However, excess Se consumption rapidly leads to toxicity; clearly, dietary Se supplementation must proceed cautiously. Regardless, over $25 \%$ of the US population use Se supplements[6] despite the probability that they have nil or detrimental effects on health.[7] Optimal dose evaluation is further complicated by the different bioactive properties of various Se compounds. Therefore, it is critical that we develop a complete in vivo understanding of Se metabolism, benefits and toxicity to provide certified advice on the appropriate human dietary intake of this biologically active trace element.

We have applied the synchrotron-based methods, X-ray fluorescence (XRF) imaging and X-ray absorption spectroscopy (XAS) to track the chemistry of selenium species in both cell culture and animal tissues under both normal physiological and disease model settings. [8] This reveals distinct fates for amino acid forms which can be related to their observed biological effect, $[9,10]$ a reductive metabolism for selenite[11] linked to delayed production of superoxide radical ions[12] and interactions with copper biochemistry,[13] as well as the discovery of a role for selenium in female reproductive function.[14] In several of these cases, the chemistry of selenium in intact tissue samples provides detail about the redox processes that are involved, while in others, redistribution of other heavy elements is informative. We are then able to link these observations to more traditional biochemical data, which provides valuable context.

As a result of this work, we formulated a hypothesis that, if correct, could reduce the increased incidence of miscarriage and birth defects in older women. The hypothesis was formed as a direct result of XRF imaging of bovine ovary tissues, which showed that selenium was transiently recruited to granulosa cells in ovarian follicles just prior to ovulation, and further biochemical studies, indicating that it was in response to a need for redox stress management. These findings suggest that oocytes in older women are exposed to greater levels of oxidative stress as a result of the aging of ovarian antioxidant defense mechanisms, and that the resulting oxidative damage to the egg is a cause of fertility issues. Prior theories had assumed that the oocytes themselves were degrading with age. If the hypothesis is true, it provides the opportunity to improve fertility in older women by boosting antioxidant defense in the ovary. Up-todate results on research pursuing this opportunity will be covered in the presentation. 
References:

[1] MP Rayman, Lancet 356 (2000), p. 233.

[2] VM Labunskyy, DL Hatfield and VN Gladyshev, Physiol Rev 94 (2014), p. 739.

[3] CM Weekley and HH Harris, Chemical Society Reviews 42 (2013), p. 8870.

[4] MP Rayman, Lancet 379 (2012), p. 1256.

[5] JC McCann and BN Ames, FASEB J 25 (2011), p. 1793.

[6] Center for Disease Control and Prevention, National Center for Health Statistics - National Health and Nutrition Examination Survey., http://www.webcitation.org/6ZVxsoxgr (accessed January 9, 2019).

[7] MP Rayman et al., Free Radical Biology and Medicine 127 (2018), p. 46.

[8] CM Weekley et al., Nutrients 5 (2013), p. 1734.

[9] CM Weekley et al., Biochemistry 51 (2012), p. 736.

[10] CM Weekley et al., Biochemistry 50 (2011), p. 1641.

[11] CM Weekley et al., Journal of the American Chemical Society 133 (2011), p. 18272.

[12] CM Weekley et al., Journal of Biological Inorganic Chemistry 19 (2014), p. 813.

[13] CM Weekley et al., Metallomics 6 (2014), p. 1602.

[14] MJ Ceko et al., Metallomics 7 (2015), p. 71.

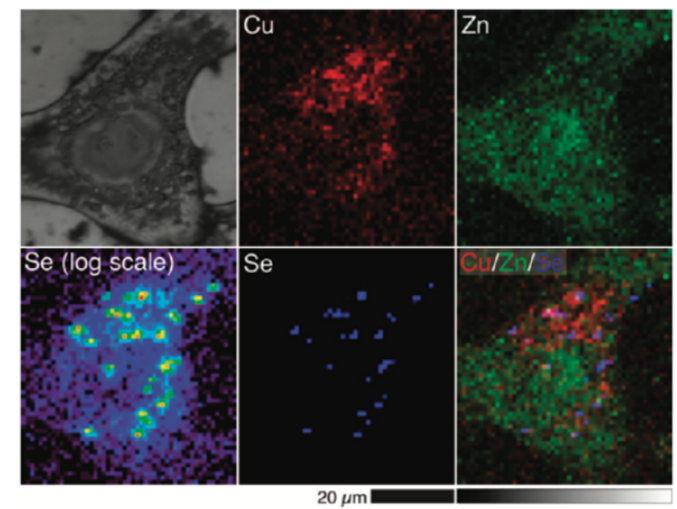

Figure 1. Optical micrograph (top left) and scattered X-ray (XS) and XRF elemental distribution maps of $\mathrm{Cu}, \mathrm{Zn}$, and $\mathrm{Se}$ (log scale and linear scale) of an A549 cell treated with $5 \mu \mathrm{M}$ selenite for $24 \mathrm{~h} . \mathrm{Cu}$ (red), Zn (green), and Se (blue) maps are overlaid to show the colocalization of the elements. Reprinted from 11 and reproduced by permission of the American Chemical Society.
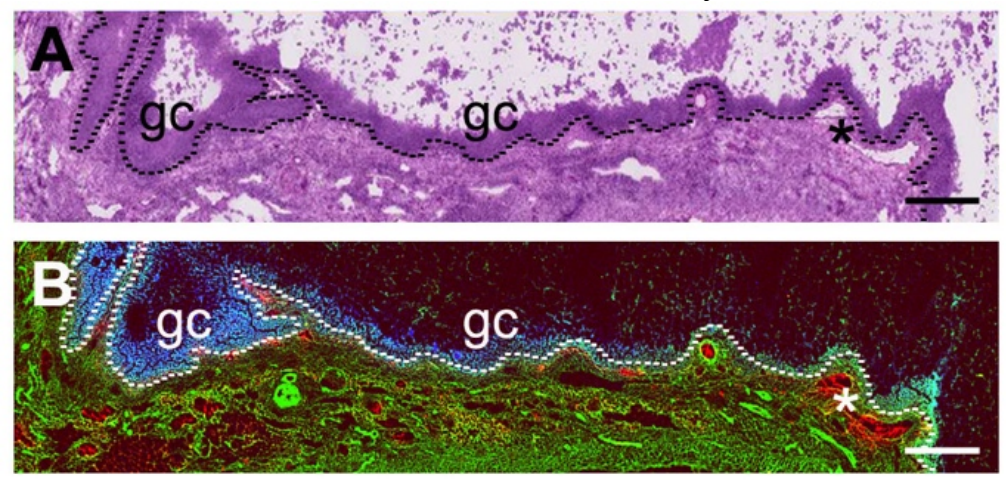

Figure 2. Localization of $\mathrm{Zn}, \mathrm{Fe}$ and $\mathrm{Se}$ in a large bovine ovarian antral follicle. (a) H\&E stained serial section of a $15 \mathrm{~mm}$ diameter healthy follicle. (b) The corresponding RGB image was generated from XRF elemental distribution maps and depicts the distribution of $\mathrm{Zn}$ (green), Fe (red) and Se (blue). * indicates vasculature; ----- indicates the separation between granulosa layer and the thecal interna; gc indicates granulosa cells. Scale bar: $500 \mu \mathrm{m}$. Reprinted from 14 and reproduced by permission of The Royal Society of Chemistry. 